Jeronimo Sirotheau de Almeida Eichler

\title{
Uma Arquitetura para Inferência de Atividades de Usuário de Computação Móvel
}

DISSERTAÇÃO DE MESTRADO

DEPARTAMENTO DE INFORMÁTICA

Programa de Pós-Graduação em Informática 


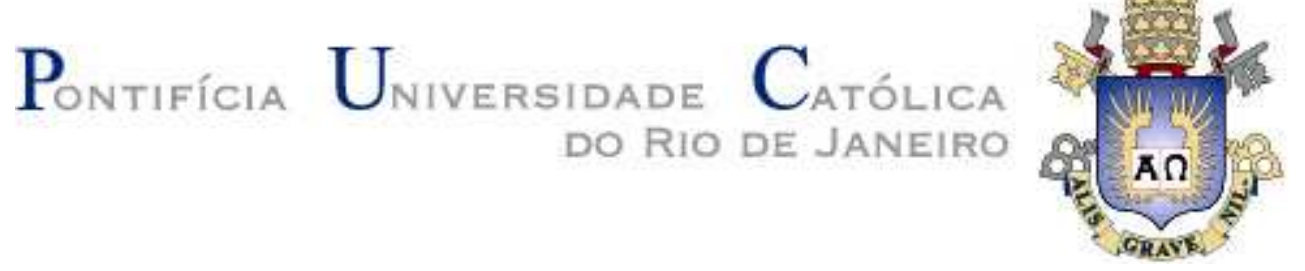

Jeronimo Sirotheau de Almeida Eichler

Uma Arquitetura para Inferência de Atividades de Usuário de Computação Móvel

\section{Dissertação De Mestrado}

Dissertação apresentada como requisito parcial para obtenção do título de Mestre pelo Programa de PósGraduação em Informática da PUC-Rio.

Orientadora: Prof ${ }^{a}$ Karin Koogan Breitman

Rio de Janeiro

Outubro de 2011 


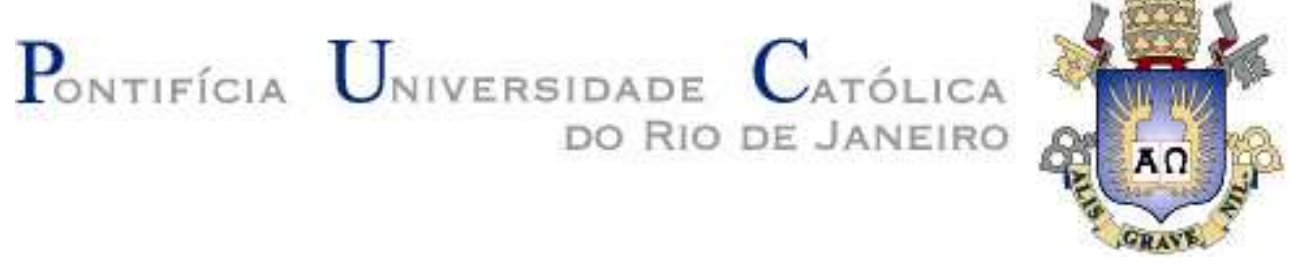

Jeronimo Sirotheau de Almeida Eichler

\section{Uma Arquitetura para Inferência de Atividades de Usuário de Computação Móvel}

Dissertação apresentada como requisito parcial para obtenção do título de Mestre pelo Programa de Pós-Graduação em Informática da PUC-Rio. Aprovada pela Comissão Examinadora abaixo assinada.

Prof ${ }^{a}$ Karin Koogan Breitman Orientadora

Departamento de Informática — PUC-Rio

Prof ${ }^{\text {a }}$ Vera Maria Benjamim Werneck UERJ

Prof. José Viterbo Filho UFF

Prof. José Eugenio Leal Coordenador do Centro Técnico Científico da PUC-Rio 
Todos os direitos reservados. É proibida a reprodução total ou parcial do trabalho sem autorização da universidade, do autor e do orientador.

Jeronimo Sirotheau de Almeida Eichler

Graduou-se em Bacharelado em Informática pela Universidade Estadual do Rio de Janeiro (UERJ) em Dezembro de 2008. Tem experiência na área de Ciência da Computação, com ênfase em Desenvolvimento de Sistemas para Internet e Aplicativos Móveis.

Ficha Catalográfica

Eichler, Jeronimo Sirotheau de Almeida

Uma arquitetura para Inferência de atividades de usuário de computação móvel / Jeronimo Sirotheau de Almeida Eichler ; orientadora: Karin Koogan Breitman. 2011.

67 f. : il. (color.) ; $30 \mathrm{~cm}$

Dissertação (mestrado)-Pontifícia Universidade Católica do Rio de Janeiro, Departamento de Informática, 2011.

Inclui bibliografia

1. Informática - Teses. 2. Inferência de atividades. 3. Ambientes inteligentes. 4. Computação ubíqua. 5. Computação móvel. 6. Ambientes sensíveis ao contexto. I. Breitman, Karin Koogan. II. Pontifícia Universidade Católica do Rio de Janeiro. Departamento de Informática. III. Título. 


\section{Agradecimentos}

Primeiramente, gostaria de agradecer a minha orientadora, Professora Karin Koogan Brietman, pelo seu conhecimento técnico, carinho, paciência e por conseguir sempre me ajudar quando foi preciso.

Gostaria de agradecer também ao Professor Markus Endler com quem aprendi muito e que contribuiu diretamente na definição do tema desta dissertação.

Agradeço aos meus familiares. Sem toda a criação que me deram e o esforço dedicado em toda a minha vida, eu jamais teria chegado aqui. Agradeço ao meu irmão, Bruno, por estar sempre ao meu lado.

À CAPES, pelo financiamento desta pesquisa. 


\section{Resumo}

Eichler, Jeronimo Sirotheau de Almeida; Breitman, Karin Koogan. Uma Arquitetura para Inferência de Atividades de Usuário de Computação Móvel. Rio de Janeiro, 2011. 67p. Dissertação de Mestrado Departamento de Informática, Pontifícia Universidade Católica do Rio de Janeiro.

A computação ubíqua aliada ao avanço tecnológico de sensores definiu um novo cenário no qual a integração de diversos recursos computacionais pode contribuir para que um conjunto de serviços e funcionalidades esteja disponível ao usuário sempre que necessário. Um subconjunto desta área é de sistemas sensíveis às atividades realizadas por seus usuários, isto é, sistemas que utilizam informações sobre o que o usuário está fazendo. Nesses sistemas, mecanismos de inferência são essenciais para reconhecer ações do usuário e permitir que o comportamento do sistema se adapte a estas ações. Entretanto, como esses ambientes são caracterizados por uma elevada troca de informações, o desenvolvimento deste tipo de sistema não é uma tarefa trivial e possui como desafios: privacidade, desempenho, complexidade e ambigüidade das informações coletadas. O objetivo deste trabalho é propor uma arquitetura para sistemas de inferências de atividades do usuário. Para atingir esse objetivo, definimos um conjunto de componentes que representam funções especificas do processo de inferência. Com o objetivo de analisar a viabilidade da arquitetura proposta, desenvolvemos, avaliamos e relatamos um protótipo de sistema baseado na arquitetura.

\section{Palavras-chave}

Inferência de Atividades; Ambientes Inteligentes; Computação Ubíqua; Computação Móvel; Ambientes Sensíveis ao Contexto. 


\section{Abstract}

Eichler, Jeronimo Sirotheau de Almeida; Breitman, Karin Koogan (Advisor). An Architecture for Inference of Activities of Mobile Computing Users. Rio de Janeiro, 2011. 67p. MSc. Dissertation Departamento de Informática, Pontifícia Universidade Católica do Rio de Janeiro.

The ubiquitous computing combined with the advance of sensor technology creates a scenario in which the integration of several computing resources is used to keep a set of services and features available to the user whenever necessary. A particular trend in this area is the activity based systems, i.e., systems that are aware of the activity played by the user. In these systems, inference engine is essential to recognize user's actions and allow the systems to adapt its behavior according to user's actions. Though, the development of this type of systems is not a trivial task as the high rate of information exchanged brings challenges related to privacy, performance and information management. In this work we propose an architecture for activity inference systems. To achieve this goal, we define a set of components that perform important roles in the inference process. Finally, to show the feasibility of this approach, we designed, implemented and evaluated a system based on the proposed architecture.

\section{Keywords}

Activity Inference; Smart Spaces; Ubiquitous Computing; Mobile Computing; Context-Aware Computing. 


\section{Sumário}

1 Introdução 12

1.1. Motivação 13

1.2. Contribuições 14

1.3. Divisão do Trabalho 14

2 Embasamento Teórico 15

2.1. Natureza do projeto 15

2.2. Inferência de atividades baseada em sensores 16

2.2.1. Sensores de localização 17

2.2.2. Sensores de movimentação 18

2.2.3. Sensores de identificação 20

2.2.4. Sensores de vídeo 20

2.3. Processo de inferência $\quad 21$

2.4. Analise dos projetos 22

2.4.1. Projetos de Prova de Conceito 22

2.4.2. Ferramentas/Aplicação 23

2.5. Tabela Comparativa 25

3 Trabalhos Relacionados $\quad 27$

3.1. P2P-DR - [04] 27

3.2. OWL-SF - [33] 27

3.3. DRAGO - [44] 28

3.4. Gaia - [39] 29

4 Arquitetura Proposta 30

4.1. Camada de fatos 31

4.2. Modelo de atividades 33

4.3. Regras de Negócio 35

4.4. Processador 37 
$\begin{array}{ll}5 \text { Ferramenta } & 39\end{array}$

5.1. Cenário do estudo de caso 39

5.2. Sistema Colossus - Diagrama de Classes 40

5.3. Sistema Colossus - Interface Gráfica 43

5.3.1. Cadastrar Usuário 44

5.3.2. Cadastrar Sala $\quad 45$

5.3.3. Excluir Sala 46

5.3.4. Conceder acesso a Agenda 46

$\begin{array}{ll}\text { 5.3.5. Consultar atividade } & 47\end{array}$

5.4. Sistema Colossus - Sensores 48

5.5. Sistema Colossus - Camada de Fatos 48

5.6. Sistema Colossus - Modelo de Atividades 49

5.7. Sistema Colossus - Regras de Negócio 50

5.8. Exemplo de iteração 52

5.9. Lições Aprendidas $\quad 57$

6 Conclusões $\quad 58$

6.1. Contribuições $\quad 59$

6.2. Limitações 60

6.3. Trabalhos Futuros 60

$\begin{array}{ll}7 \text { Referências Bibliográficas } & 62\end{array}$ 


\section{Lista de figuras}

Figura 1 - Informação de localização 18

Figura 2 - Informações de localização e deslocamento 19

Figura 3 - Arquitetura 31

Figura 4 - Visão do Processador, Regras de Negócio e Modelo de Atividades 33

Figura 5 - Máquina de estados de fazer café $\quad 34$

Figura 6 - Exemplo de Ontologia $\quad 35$

Figura 7 - Processamento sob demanda $\quad 37$

Figura 8 - Processamento em Ciclos 38

Figura 9 - Sistema Colossus - Visão de Pacotes 41

Figura 10 - Casos de Uso Sistema Colossus 43

Figura 11 - Tela Cadastrar Usuário 44

Figura 12 - Tela Cadastrar Sala $\quad 45$

Figura 13 - Tela Excluir Sala 46

Figura 14 - Tela Autorizar Acesso 46

Figura 15 - Tela Permissão Contas Google $\quad 47$

Figura 16 - Tela Consultar Atividades 47

Figura 17 - Modelo de Atividades 49

Figura 18 - Informações coletadas da Google Agenda 52

Figura 19 - Multi-Processamento na Arquitetura 61 


\section{Lista de tabelas}

Tabela 1 - Projetos Correlatos $\quad 15$

Tabela 2 - Tipos de Sensores 16

Tabela 3 - Análise de Projetos de Inferência de Atividades 25

Tabela 4 - Tabela de Estados 50

Tabela 5 - Tabela de Estimulo $\quad 50$

Tabela 6 - Regras de Inferência $\quad 51$

Tabela 7 - Informações de Contexto Coletadas 53

Tabela 8 - Inferência de Atividades $\quad 54$

Tabela 9 - Atividades Realizadas $\quad 56$ 